\title{
In Situ Environmental Transmission Electron Microscopy of Ice Nucleation
}

\author{
Lyle M. Gordon, Libor Kovarik
}

Environmental Molecular Spectroscopy Laboratory, Pacific Northwest National Laboratory, 3335 Innovation Blvd. Richland, WA 99354

Heterogeneous ice nucleation on atmospheric aerosols results in cloud formation and represents one of the largest uncertainties in predicting climate. Elucidating the fundamental physicochemical and materials properties that result in highly active ice nuclei requires detailed observation of the ice nucleation process of model substrates in situ. Environmental transmission electron microscopy (TEM) represents a suitable tool for high-resolution analysis of nucleation in situ. Unlike a conventional TEM, gas can be introduced to the sample to simulate the reaction conditions of interest.

Amorphous ice is frequently utilized by transmission electron microscopy (TEM) to study the structure of biological molecules frozen in a vitreous ice layer at liquid nitrogen temperature. Recently, Kobayashi et al. achieved atomically resolved image of ex situ and in situ nucleated ice nanocrystals using TEM [1-2]. These experiments demonstrate the possibility of conducting in situ ice nucleation in the TEM, however, the work presented was limited to cryogenic $(<100 \mathrm{~K})$ temperatures and high vacuum conditions in a conventional TEM. In situ investigation of atmospheric nucleation on natural aerosols requires simulation of atmospherically relevant conditions, specifically temperatures between $200-273 \mathrm{~K}$ and water vapor partial pressures in the range of $10^{-3}-10 \mathrm{mbar}$.

I will present results from initial experiments performed in an FEI Titan ETEM. Experiments were performed with a custom-built gas mixer and a commercially available liquid nitrogen cooled cryogenic TEM holder equipped with a resistive heater and temperature controller. To study atmospheric heterogeneous ice nucleation processes, model aerosol nanoparticles were dispersed on an amorphous carbon substrate. The substrate was subsequently cooled and water vapor was introduced to the chamber. Multiple images were acquired throughout the nucleation process (Figure 1). Nucleation was frequently observed within high curvature regions between the nanoparticle and the substrate likely due to the Kelvin effect. This observation could be evidence that depositional ice nucleation (vapor-solid) is in fact a two-step process where liquid water condenses in high-curvature nanopores below bulk water saturation and then freezes by homogenous or heterogeneous nucleation [1-4].

\section{References:}

[1] Kobayashi, K. et al, Physical Review Letter 106 (2011), p. 1.

[2] Kobayashi, K. et al, Chemical Physics Letters 106 (2012), p. 9.

[3] Marcolli, C., Chemical Physics Letters 14 (2014), p. 2071.

[4] The research was performed using EMSL, a DOE Office of Science User Facility sponsored by the Office of Biological and Environmental Research and located at Pacific Northwest National Laboratory. 

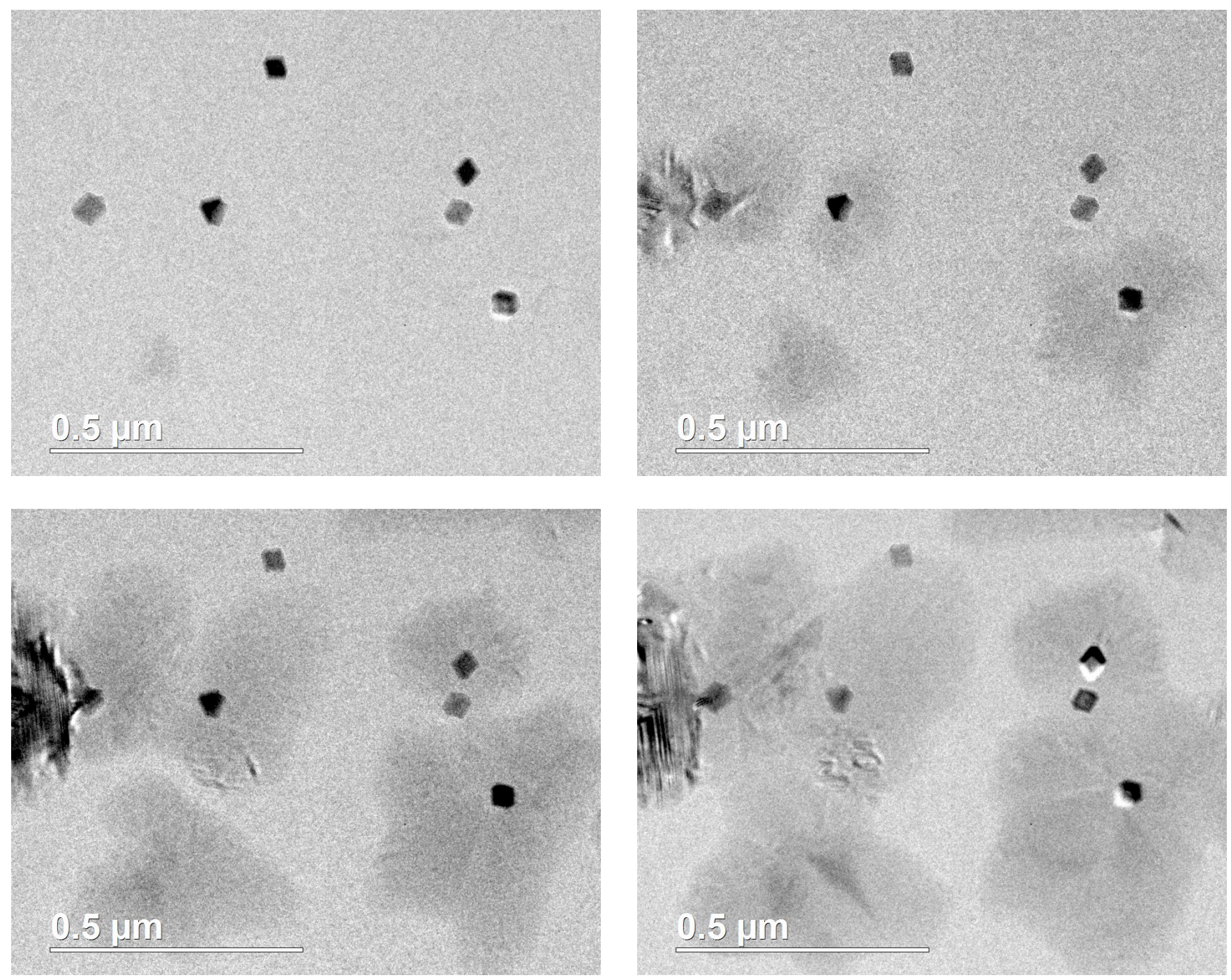

Figure 1. Selected frames from a movie of in situ nucleation of ice crystals on hematite nanoparticles supported on an amorphous carbon film. Substrate temperature: $\sim 200 \mathrm{~K}, \mathrm{H}_{2} \mathrm{O}$ partial pressure during growth: $\sim 10^{-2}$ mbar. 